\title{
Limites de l'application de la méthode de conservation du lait cru par le système lactoperoxydase en Afrique de l'Ouest
}

\author{
F. Lhoste ${ }^{1}$
}

\author{
Mots-clés \\ Lait - Collecte - Préservation - Codex \\ Alimentarius - Afrique occidentale.
}

\begin{abstract}
Résumé
Dans cette étude ont été analysées les raisons pour lesquelles l'adoption de la méthode de conservation du lait cru par le système lactoperoxydase (s-LP) par les pays africains a donné des résultats mitigés en dépit de l'intérêt qu'elle suscitait. L'analyse a été basée sur l'évaluation de l'impact du projet Lait de brousse lancé en 2001 par la FAO et s'est appuyée sur la mission d'évaluation du projet, menée dans trois pays d'Afrique de l'Ouest, ainsi que sur l'atelier régional tenu au Niger en 2004. La principale limite à l'adoption de cette méthode était liée au manque de structuration professionnelle des filières laitières locales, nécessaire à l'utilisation de la méthode s-LP.
\end{abstract}

\section{INTRODUCTION}

Dans les pays qui possèdent une industrie laitière avancée, le refroidissement sauvegarde la qualité bactérienne du lait cru et en empêche la détérioration. Toutefois, le refroidissement est rarement une technique de conservation applicable pour les petits producteurs des zones de production laitière des pays en développement en raison de l'absence d'un système d'alimentation électrique fiable ou à cause de contraintes économiques $(11,13,15)$.

Le programme Lait de brousse (ci-après noté « $\mathrm{LdB} »)$, visant à informer les gouvernements d'Afrique de l'Ouest de l'existence d'une méthode de conservation du lait alternative à la réfrigération, a été lancé en 2001 par la FAO et financé par le gouvernement français. Des démonstrations de cette méthode de conservation $\mathrm{du}$ lait cru par le système lactoperoxydase (s-LP) ont été réalisées dans une dizaine de pays de la sous-région.

Beaucoup de pays d'Afrique de l'Ouest tentent aujourd'hui de renforcer et de structurer leurs filières laitières et de réduire le volume d'importation de lait et de produits laitiers. De ce fait, de nombreuses petites industries de transformation de lait ont émergé et tentent de développer leur propre système de collecte du lait. Malgré ces

1. Ecole nationale d'industrie laitière de Besançon, 25620 Mamirolle, France. Tél. : +330633794974 ; e-mail : lhoste99@yahoo.fr tendances, le système s-LP n'est toujours pas beaucoup utilisé dans la sous-région.

Incontestablement, le développement de la méthode de conservation du lait cru par l'activation du système lactoperoxydase $(5,6)$ n'a pas atteint les objectifs attendus qui prévoyaient une adoption et une dissémination de la méthode dans la grande majorité des pays du Sud après la phase de démonstration (13). Ces résultats n'ont pu être obtenus malgré l'adhésion au programme mondial de lactoperoxydase de plus de 80 pays membres de la FAO et les démonstrations effectuées dans plus de 40 pays en Afrique, Asie du Sud Est, Amérique centrale et Amérique latine.

L'Afrique de l'Ouest ne fait pas exception à la règle, malgré l'émergence récente de petites et moyennes structures qui s'organisent autour de l'activité laitière et utilisent à divers degrés le lait local. Dans l'ensemble des pays de la sous-région, l'engouement des entreprises artisanales de collecte et de transformation de lait est à souligner avec des résultats variés sur le plan économique (2). Pour diverses raisons, telles que le prix des matières premières ou la disponibilité des équipements, les entreprises artisanales et industrielles ont pris l'habitude, dans le passé, de travailler essentiellement avec de la poudre de lait ; elles ont fait preuve de peu d'initiatives alternatives. Finalement les stratégies mises en œuvre l'ont été au détriment du développement des filières laitières locales $(13,15)$. 
La méthode s-LP apparaît pourtant comme une solution alternative et/ou complémentaire à la réfrigération ; elle peut présenter des avantages significatifs en attendant que les pays concernés se dotent d'infrastructures laitières organisées, et en répondant aux besoins des producteurs ruraux, des transformateurs et des consommateurs urbains. L'entrée spécifique technique et sociale représentée par la collecte du lait à travers le $\mathrm{LdB}$ a présenté l'avantage d'aborder directement les problématiques de l'organisation des unités artisanales laitière en Afrique de l'Ouest (13) et du développement de lait de chamelle en Afrique (7). Ce programme avait pour principal objectif d'informer les pays d'Afrique de l'Ouest sur l'intérêt et la pratique de cette méthode alternative à la réfrigération pour la conservation du lait cru. Les pays prioritaires soutenus par ce programme ont été le Bénin, le Burkina Faso, le Cameroun, la Côte d'Ivoire, la Guinée, le Mali, la Mauritanie, le Niger, le Sénégal, le Tchad et le Togo.

\section{METHODE}

\section{Principe du système lactoperoxydase}

La lactoperoxydase est une enzyme endogène et abondante dans le lait cru. Les deux composants essentiels au fonctionnement du s-LP sont le peroxyde d'hydrogène et le thiocyanate, naturellement présents dans le lait à des concentrations variables selon l'alimentation, l'espèce et la race de l'animal. La lactoperoxydase enzymatique participe à la production d'un composé antibactérien naturellement actif dans divers fluides corporels comme les larmes, la salive et les sucs gastriques. Ce composé participe, parmi d'autres systèmes antibactériens du lait, à la protection bactérienne des jeunes mammifères durant l'ingestion et la digestion du lait maternel $(5,6)$.

Dans le lait cru, l'activité antibactérienne est faible et ne dure que deux heures après la traite. La méthode de réactivation du s-LP consiste à ajouter artificiellement les deux composants d'activation du système pour prolonger de sept à huit heures la durée de conservation du lait cru à une température ambiante de $30^{\circ} \mathrm{C}(5,6)$.

\section{Intérêt pratique de la méthode}

Pour une application correcte de cette méthode, les producteurs, ou plus souvent les femmes responsables de la gestion du lait, doivent transporter rapidement le lait destiné à la vente jusqu'à un point de collecte situé à proximité du lieu de la traite.

Grâce au traitement s-LP sur le lait de mélange au point de collecte, le lait peut alors être transporté par différents moyens pendant sept à huit heures, jusqu'à un centre de traitement $(5,7,9,11,13)$. Lorsque sont mis en place des points de collectes fonctionnels proches des lieux de production, l'augmentation des volumes commercialisés réalisée par le s-LP peut ainsi participer à améliorer significativement le revenu généré au niveau des petits producteurs.

Il est essentiel que la qualité du lait cru soit la meilleure possible car le s-LP n'a pas pour effet d'améliorer la qualité hygiénique du lait. L'amélioration de l'hygiène en production laitière est donc complémentaire à l'application du s-LP pour assurer la conservation du lait. C'est pour cela que dans les différents pays participants, durant les missions du LdB, une attention particulière a été portée sur la sensibilisation de la qualité hygiénique du lait et sa manipulation. Plus particulièrement les formations ont concerné les bonnes pratiques d'hygiène et de la traite, le transport et le traitement du lait ainsi que la transformation. Au niveau mondial, le s-LP reste aujourd'hui utilisé de façon marginale en Afrique, Asie et Amérique latine ; le seul pays où son utilisation est reconnue à une échelle nationale est Cuba (16) .

\section{Méthode d'évaluation de l'impact du projet}

La méthode d'évaluation de l'impact du LdB a consisté à comparer les résultats du programme dans chacun des pays participants avec les résultats attendus en termes de connaissance de la méthode s-LP et d'adoption. Cela a pu être réalisé lors d'un atelier de restitution du programme qui réunissait en 2004 l'ensemble des pays participants au Niger (9) et durant les missions d'évaluation du projet en 2006 au Niger, Bénin et Sénégal (12). Les interventions dans chaque pays se sont situées à deux niveaux : un niveau national pour l'information des services étatiques et un niveau local pour les démonstrations et formations des groupements laitiers féminins et des associations d'éleveurs.

\section{RESULTATS DU PROGRAMME}

\section{Impacts du programme Lait de brousse}

Le LdB a permis une large sensibilisation des différents intervenants de la filière sur la structuration et l'organisation d'un secteur laitier qui intègre aussi bien les producteurs, les transformatrices et les revendeuses de lait que les services étatiques de vulgarisation agricole et de santé animale, ou bien encore les consommateurs. La sensibilisation sur l'amélioration de la qualité hygiénique du lait et sa préservation par la méthode s-LP, point phare de ce programme, a eu un impact important auprès des producteurs, des groupements féminins et des services de vulgarisation étatiques. Les nombreuses formations, majoritairement suivies par des femmes, ont permis aux participants d'échanger leur expérience, d'exposer leurs pratiques, mais aussi d'apprendre et de découvrir des moyens et des techniques appropriés afin de réduire les pertes de lait de la ferme, jusqu'au niveau des consommateurs.

Le programme a rempli ses objectifs en termes de diffusion de l'information technique et logistique nécessaire pour gérer une activité laitière intégrée et contribuer à l'amélioration de la qualité et des volumes de lait collectés et commercialisés dans de nombreux bassins laitiers.

La technologie s-LP est maintenant bien connue, ses modalités d'emploi ont été bien définies, seul l'aspect législatif reste encore à adapter en fonction des choix, des lois et des règles de chaque pays.

Un autre impact important du programme a été l'identification des défaillances à la construction de la filière du lait local qui a conduit à l'accompagnement des partenaires gouvernementaux pour le renforcement des mesures gouvernementales, nationales et régionales d'appui au sous-secteur laitier. L'approche régionale devrait permettre d'harmoniser et de renforcer les systèmes d'assurance qualité, l'établissement et le contrôle des normes ainsi que les accords marchands et douaniers laitiers dans la sous-région. La salubrité des produits laitiers d'origine locale ou importés devrait s'intégrer dans une dynamique plus large d'amélioration de la salubrité de l'alimentation des populations africaines et de la santé publique.

Enfin, le programme a eu un impact certain sur la réduction des pertes, mais aussi sur l'amélioration de la qualité hygiénique du lait et des produits laitiers, la santé animale et l'organisation des producteurs qui représentent des champs d'actions prioritaires des projets de la FAO. En effet, des études de cas récentes (8) menées en Asie et en Afrique de l'Est montrent que la collecte locale de 100 litres de lait par jour est susceptible d'engendrer la création de cinq emplois dans le secteur (transport, transformation, vente).

On peut conclure que le programme a eu un sérieux impact à un niveau national et local en termes d'information de cette méthode alternative à la réfrigération ; en revanche, l'impact reste faible en 
termes de dissémination de l'utilisation du s-LP, comme escompté dans les objectifs du projet.

\section{Efficacité de la méthode s-LP, intérêt des démonstrations}

Bien que l'efficacité du traitement ne soit plus à prouver, ces démonstrations ont permis de faire connaître aux acteurs des filières laitières locales l'intérêt économique d'une telle méthode qui permet d'augmenter la conservation du lait cru avant son acidification et donc d'offrir la possibilité de commercialiser des volumes de lait plus important (5).

Au Niger (13), malgré les conditions extrêmes de la température de l'air et du lait et les très mauvaises conditions d'hygiène de la traite et du transport du lait cru, le traitement s-LP a permis de stabiliser l'évolution bactérienne du lait pendant quatre ou cinq heures alors que sa température était comprise entre 33 et $35^{\circ} \mathrm{C}$. Ces résultas proviennent de la mission de la FAO en 2002 au Niger où cinq sites de démonstration avaient été mis en place : le centre de multiplication du bétail de Toukounous, situé à $212 \mathrm{~km}$ au nord est de Niamey et auprès de quatre collecteurs privés, situés dans un rayon de $40 \mathrm{~km}$ autour de Niamey, livrant leur lait aux laiteries Niger-lait et Solani.

Au Sénégal, la qualité du lait a été stabilisée pendant sept heures, durée largement suffisante pour organiser sa commercialisation dans des conditions acceptables (13). Les démonstrations ont été réalisées dans les régions de Tambacounda à $480 \mathrm{~km}$ au sud est de Dakar, et de Saint-Louis à $270 \mathrm{~km}$ au nord de Dakar. Elles ont permis la formation des personnes impliquées dans la filière laitière, en insistant sur le fait que le recours au traitement s-LP ne permettait pas de se soustraire aux règles impératives d'hygiène. Elles ont également montré qu'il était très important de mettre à profit la présence des éleveurs et des productrices ayant livré leur lait au point de collecte s-LP pour les sensibiliser sur l'importance des points suivants : le respect des règles d'hygiène concernant la traite et la vaisselle laitière, le refroidissement du lait après la traite, et enfin la nécessité de livrer leur lait au point de collecte dans les plus brefs délais et en respectant les règles d'hygiène de manipulation du lait frais.

Les séances de démonstration ont montré aux opérateurs de la filière l'efficacité et l'intérêt économique que pouvait représenter le traitement s-LP pour la conservation du lait cru. Elles ont également relevé l'importance qu'il y a lieu d'accorder à l'hygiène du lait depuis la traite jusqu'à la transformation. Les tests de filtration qui témoignaient de la propreté macroscopique du lait ont permis de faire visualiser aux éleveurs les efforts importants restant à faire en matière d'hygiène dans la manipulation du lait.

Des pertes de lait ont été identifiées à différents niveaux de la production en passant par la transformation et jusqu'à la commercialisation. Les pertes étaient majoritaires au niveau des unités de production villageoise par manque de moyens de conservation du lait.

\section{Forces et faiblesses du programme}

Ce paragraphe synthétise les principales remarques issues d'ateliers de restitution nationaux et régionaux réalisés lors des missions relatives à ce programme pour le développement des filières lait en Afrique de l'Ouest $(9,12,13)$. Les forces résidaient dans l'adhésion des partenaires (producteurs, laiteries, structures encadrement), la volonté politique, la simplicité et efficacité de la technique présentée, l'augmentation actuelle des petites et moyennes laiteries en nombre et en capacité, et l'intérêt important des producteurs. Les faiblesses portaient sur l'insuffisance des moyens techniques et logistiques pour poursuivre les activités, l'insuffisance de dialogue et de concertation entre producteurs et industries (partage des coûts), l'absence de financement relais pour le programme, et le manque de structuration actuelle des filières et de l'interprofession laitière.

Des solutions alternatives pourraient notamment intégrer, d'une part, une meilleure contribution des projets d'appui à la filière lait dans la mise en œuvre des démonstrations, à travers la prise en charge des interventions dans leur zone et/ou l'acquisition de matériel technique, et, d'autre part, la formation du personnel d'encadrement au niveau décentralisé pour en faire des relais du comité au niveau régional et local.

\section{DISCUSSION ET PROPOSITIONS}

\section{Ambiguité dans la législation : un frein à l'adoption du s-LP}

La directive pour l'utilisation d'une méthode alternative de conservation de lait basée sur un complexe antibactérien enzymatique du lait a été approuvée par le comité d'experts de la FAO et de l'OMS sur les additifs en 1989, et par la commission du Codex Alimentarius en 1991, stipulant que cette méthode était d'une utilisation sûre et sans danger (6). Dans les années qui suivirent, de 1995 à 2002, sont apparues par l'intermédiaire de différentes commissions du Codex Alimentarius, des restrictions concernant la commercialisation à un niveau international des produits laitiers dont le lait a initialement subi le traitement s-LP. Cela est logiquement considéré par certains pays comme une contrainte restrictive importante qui freine l'adoption de la méthode.

Fin 2005, une revue d'experts a été organisée par la FAO et l'OMS, sur les bénéfices et risques du système lactoperoxydase (10); elle a notamment recommandé de lever la restriction sur le commerce international. La commission du Codex Alimentarius n'a cependant pas suivi cette recommandation. Il n'existe donc pas, à ce jour, d'évolution de la législation concernant les produits laitiers ayant subi initialement le traitement s-LP.

Cette évolution des directives pourrait pourtant permettre une certaine relance du processus d'adoption technique et législative en Afrique de l'Ouest mais également en Afrique de l'Est. Il est difficile d'expliquer le bien-fondé de cette restriction si ce n'est en envisageant sa corrélation à des enjeux économiques importants.

\section{Systèmes d'organisations paysannes peu préparés à accepter la méthode s-LP}

Dans les pays qui possèdent une industrie laitière avancée, le refroidissement sauvegarde la qualité bactérienne du lait cru et en empêche la détérioration. Toutefois, le refroidissement est rarement une technique de conservation applicable pour les petits producteurs des zones de production laitière des pays en développement en raison de l'absence d'un système d'alimentation électrique fiable ou à cause de contraintes économiques $(11,13,15)$.

Selon les hypothèses $\mathrm{du} \mathrm{LdB}$, la méthode pourrait permettre d'augmenter les périmètres des zones de collecte du lait et ainsi de désenclaver des bassins de production laitière éloignés des centres urbains. Les bassins de production situés en zone périurbaine ou intra-urbaine (4) n'ont en revanche pas forcément besoin du recours à cette méthode. Le manque d'organisation des systèmes laitiers ouest africains ne permet pas toujours l'utilisation rationnelle du s-LP dans le respect des directives en vigueur. A part quelques exemples marginaux tels que la laiterie Tivisky en Mauritanie, 
il est actuellement difficile d'identifier des sociétés industrielles ou des coopératives laitières d'envergure nationale ou régionale capables de traiter des volumes de lait local conséquents, de développer un système de collecte organisé et de commercialiser par la suite ce lait.

\section{Mise sur le marché compliquée des activateurs s-LP}

L'application du s-LP est théoriquement bon marché. Pour permettre sa diffusion, la FAO, en accord avec les différents fournisseurs d'activateurs, a évalué le coût de ce traitement du lait cru à environ 0,01 \$ US par litre de lait, sans taxe. Cependant, ce coût ne prend pas en compte les taxes douanières que peuvent décider d'appliquer certains pays ainsi que les frais d'envoi des activateurs. De plus, ce type de produit peut être diffusé soit par une coopérative ou une entreprise laitière, soit par des entreprises pharmaceutiques et vétérinaires privées qui prennent naturellement une marge supplémentaire sur la vente (13). La pertinence et la rationalité économiques d'utilisation d'une méthode qui se voulait très bon marché sont alors remises en cause, et elle peut alors apparaître trop risquée pour des producteurs pour qui la vente du lait n'est pas sécurisée.

\section{Technique susceptible d'améliorer la compétitivité des filières locales}

L'activité laitière fournit aux femmes peules un revenu quotidien régulier, indispensable à la sécurité du ménage et au bien-être de la famille. Suivant les zones, les femmes ne participent pas seulement de manière directe à la production de lait mais elles sont aussi le plus souvent responsables des opérations de collecte, transformation et commercialisation des produits laitiers. Le rôle traditionnel que jouent les femmes dans le secteur laitier à petite échelle dans le monde entier et spécialement en Afrique de l'Ouest est maintenant reconnu et bien pris en considération par les programmes sectoriels d'appui au développement des filières agricoles.

Les fortes variations saisonnières sont difficilement maîtrisables et entraînent des adaptations des pratiques et des stratégies variées. En effet, la production abondante en saison des pluies entraîne une saturation du marché local, malgré l'approvisionnement de petites unités de transformation. En revanche, en saison sèche, la rareté du lait local entraîne une vente en direct et empêche l'approvisionnement en quantité des laiteries.

De façon temporaire et dans certains contextes particuliers, on conçoit que l'utilisation du s-LP pourrait participer à la réduction des pertes d'un produit aussi périssable que le lait. L'afflux massif et régulier de poudre de lait depuis une vingtaine d'années, fortement critiqué par nombre d'organisations (1), a toutefois le mérite de permettre à la fois le maintien d'une activité des entreprises laitière, de participer à l'équilibre alimentaire des populations et de préparer un avenir pour l'essor de filières laitières. En effet, les importations massives de poudre de lait ont permis une industrialisation rapide du secteur de la transformation qui pourrait à l'avenir offrir des débouchés croissants pour la production locale $(3,4)$.

Cependant la non-compétitivité actuelle des produits laitiers locaux s'explique par, d'une part, l'absence de politique laitière volontariste et, d'autre part, la présence d'une poudre de lait trop bon marché (15). Les données chiffrées présentées par Metzger et coll. (14), bien que datant d'une dizaine années, sont encore cohérentes aujourd'hui et montrent sans ambiguïté que le lait produit à partir de poudre de lait importée a un coût de revient bien inférieur au prix du lait frais local commercialisé en milieu urbain. Même en levant ces obstacles, un certain nombre de contraintes (organisationnelles, techniques, sociologiques) propres à l'émergence de filières locales pèsent sur ces dernières. Même la dévaluation du franc CFA en 1994 n'a pas permis de rendre suffisamment compétitifs les produits laitiers locaux tant les marges dégagées par les entreprises travaillant avec de la poudre de lait importée sont considérables, spécialement pour le yaourt et le lait caillé où la matière première ne représente parfois que de 10 à 25 p. 100 du prix final du produit (14).

\section{Propositions technico-économiques pour l'utilisation de la méthode s-LP}

Durant les différents ateliers réalisés dans le cadre des missions du LdB (13) et les nombreuses séances de démonstration, les pays participants ont évoqué les points rappelés ci-dessous, pour lesquels des actions devraient être engagées, qui renvoient à des responsabilités partagées :

- adapter la présentation actuelle des activateurs du système s-LP au contexte local ;

- aider les pays à se doter d'un cadre législatif et réglementaire relatif à l'utilisation du système s-LP ;

- demander au Codex Alimentarius la levée de la restriction au commerce international du lait et produits laitiers traités qui n'est scientifiquement pas fondée ;

- appuyer les dynamiques d'organisation de tous les acteurs de la filière laitière ;

- créer des centres de collecte ;

- promouvoir des conditions favorables à la transformation du lait local (fiscalité, recherche et développement, réglementation et renforcement de capacité) ;

- définir et appliquer des normes adaptées de production et de transformation ;

- créer un label pour le lait et les produits laitiers locaux ;

- promouvoir les conditions pour une participation avec concertation de tous les acteurs en vue d'assurer la sécurité sanitaire du lait et des produits laitiers ;

- mettre en place des guides de bonnes pratiques à toutes les étapes de la filière laitière de façon concertée.

\section{Principaux enseignements du programme Lait de brousse}

Les partenaires ayant pris part aux démonstrations de conservation du lait cru par activation du système lactoperoxydase ont reconnu ce dernier comme étant une méthode adaptée au contexte en Afrique de l'Ouest. L'utilisation de la méthode s-LP à plus grande échelle est envisageable à condition de mettre en place un dispositif approprié prenant en compte les principales contraintes organisationnelles, sociales et économiques ainsi que les aspects réglementaires internationaux. A l'instar d'autres transferts de technologie, la méthode peut participer ponctuellement à l'amélioration de la collecte du lait dans les zones ne possédant pas d'infrastructures de froid. Elle ne sera vraiment intéressante qu'intégrée dans un contexte plus large de formation sur les bonnes pratiques et l'hygiène de l'activité laitière.

Globalement en Afrique de l'Ouest, les législations laitières présentent des défaillances et ne correspondent pas aux normes internationales établies. Le cadre de prévention et de contrôle des denrées alimentaires d'origine animale est très peu actif et ne semble pas être une priorité pour les autorités. La faiblesse des moyens financiers, matériels et humains consacrés à la mise en place de systèmes d'assurance qualité est préjudiciable à l'accompagnement des filières des produits d'élevage, notamment pour les produits laitiers. 
Les initiatives privées et coopératives impliquées dans les activités laitières ainsi que le rôle des consommateurs urbains devraient permettre d'améliorer progressivement l'adaptation des systèmes de contrôle.

Dans l'ensemble de la sous-région, le marché est favorable au lait local : la demande en produits laitiers est en pleine expansion. Incapables de répondre à la demande en produits standardisés (lait pasteurisé ou stérilisé, lait en poudre, lait écrémé, desserts lactés, beurre pasteurisé, fromages), les petites entreprises laitières locales doivent rechercher des segments de marché spécifiques comme le fromage artisanal (par exemple le wagashi au Bénin), le lait fermenté artisanal ou encore les yaourts.

Malgré l'impact limité représenté par les unités artisanales (2, 3 ), ces dernières représentent des maillons essentiels de la filière laitière. Adaptées au contexte local, elles offrent aux éleveurs et transformatrices la possibilité d'écouler les excédents de lait et induisent chez eux de nouveaux comportements et de nouvelles pratiques. Toutefois, le nombre et la taille de ces unités de réception et de transformation du lait ne suffisent pas encore à garantir un niveau de production permettant une présence permanente de produits issus du lait local sur les marchés urbains éloignés.

\section{CONCLUSION}

L'organisation de nouveaux marchés laitiers en Afrique de l'Ouest exige la mobilisation de ressources humaines, le renforcement des capacités des acteurs et le transfert de technologies adaptées au contexte. Des innovations technologiques sont nécessaires pour garantir une meilleure qualité hygiénique des produits, tout en restant adaptées et aussi simples que possible dans le choix des équipements.

Cependant, cette organisation exige également une amélioration des systèmes de production qui doit aller de pair avec les innovations technologiques dans la filière. Pour les éleveurs, les dispositifs d'amélioration de la production doivent être rémunérateurs et sécurisants face aux risques d'investissements.

Ainsi, alors que l'utilisation de la méthode s-LP semblait pouvoir répondre à des contraintes ponctuelles de conservation d'un produit aussi périssable que le lait, le LdB n'a pas permis une diffusion rapide de la méthode en Afrique de l'Ouest. Les limites à la mise en œuvre de cette technique ont été identifiées. La principale difficulté à l'adoption de la méthode est due au manque organisationnel des filières et le peu d'intérêt des décideurs pour l'appui aux filières lait local à l'époque du projet.

Le prix des activateurs pour les utilisateurs finaux serait à mettre en corrélation avec la valorisation du litre de lait jusqu'alors sous-valorisé mais ne devrait pas représenter un problème au sein d'un système organisé. Le cadre législatif n'est que secondaire en Afrique de l'Ouest et semble avoir plus d'importance en Afrique de l'Est. Le projet a conclu sur l'importance de poursuivre le renforcement institutionnel de ces filières locales qui jouent un rôle important dans la création de richesse en zones rurales.

\section{Remerciements}

L'auteur tient à remercier particulièrement le Gouvernement français et la division de la Santé et des Productions animales de la FAO qui ont permis la réalisation financière et logistique de ce programme. Des remerciements sont également adressés à l'équipe du service de la Production animale de la FAO pour son implication tout au long de ce programme, ainsi que tous les organismes et institutions partenaires ayant participé à son achèvement. L'auteur adresse de sincères remerciements aux différents partenaires africains qui se sont impliqués et ont facilité les différentes actions du programme. Enfin de chaleureux remerciements sont adressés aux groupements laitiers féminins et aux éleveurs pour leur accueil lors des séances de démonstration, pour leur dynamisme, patience et disponibilité.

\section{BIBLIOGRAPHIE}

1. CFSI, 2006. Campagne Alimenterre - Lait - I'Europe est vache avec I'Afrique. Paris, France, CFSI. http://www.cfsi.asso.fr/

2. CORNIAUX C., DUTEURTRE G., DIEYE P.N., POCCARD-CHAPUIS R., 2005. Les minilaiteries comme modèle $d^{\prime}$ organisation des filières laitières en Afrique de l'Ouest : succès et limites. Revue Elev. Méd. vét. Pays trop., $58: 237-243$

3. DUTEURTRE G., DIEYE P.N., DIA D., 2005. Ouverture des frontières et développement agricole dans les pays de l'Uemoa. L'impact des importations de volailles et de produits laitiers sur la production locale au Sénégal. Dakar, Sénégal, Isra, 78 p. (Etudes et documents $n^{\circ} 8$ )

4. DUTEURTRE G., MEYER C., 1998. Marchés urbains et développement laitier en Afrique subsaharienne. In : Actes atelier international, Montpellier, France, 9-10 sept. 1998. Montpellier, France, Cirad-emvt, $233 \mathrm{p}$.

5. FAO., 1999. Manuel sur la lactoperoxydase pour la manutention et la conservation du lait. Programme mondial sur la lactoperoxydase. Rome, Italie, FAO, 36 p.

6. FAO, 2000. Directives sur I'application du système de la lactoperoxydase pour la conservation du lait. Codex Alimentarius. Lait et produits laitiers, vol. 12, 2 é édn. Rome, Italie, FAO, p. 129-136.

7. FAO, 2003. Lait de chamelle pour I'Afrique. Compte rendu de l'atelier sur la filière caméline en Afrique. Rome, Italie, FAO, 122 p. http://www. fao.org/ag/againfo/resources/en/publications/agapubs/ChamelleBook.pdf

8. FAO, 2004. Employment generation through small-scale dairy marketing and processing. Experiences from Kenya, Bangladesh and Ghana. Joint study ILRI Market-oriented Smallholder Dairy Project / FAO Animal Production, Health Division. Rome, Italy, FAO, 59 p. http://www. fao.org/ag/againfo/subjects/en/dairy/pubs.html

9. FAO, 2004. La collecte du lait dans les zones sans infrastructures laitières en Afrique centrale et de l'Ouest. Compte rendu atelier régional. Rome, Italie, FAO, 86 p.

10. FAO, 2005. Avantages et risques potentiels du système lactoperoxydase pour la conservation du lait cru. Rapport. Rome, Italie, FAO, 68 p. ftp://ftp.fao.org/codex/ccfh38/fh38_lpf.pdf

11. LAMBERT J.C., 1988. La transformation laitière au niveau villageois. Rome Italie, FAO, 73 p. (Etude $n^{\circ} 69$ )

12. LHOSTE F., 2006. Impact du projet Lait de brousse. Evaluation réalisée au Niger, Bénin et Sénégal. Rome, Italie, FAO, 75 p.

13. LHOSTE F., RAMET J.P, SOUKEHAL A.H., 2001-2005. Le Lait de brousse. L'organisation de la collecte de lait dans les zones sans infrastructures laitières en Afrique de l'Ouest. Rapports de mission dans les pays participants et comptes rendus d'ateliers dans le cadre du programme Lait de brousse. Rome, Italie, FAO.

14. METZGER R., 2003. Approche économique de la filière laitière en Guinée - Appui à I'amélioration de la collecte, de la transformation et de la commercialisation du lait et des produits laitiers en Guinée. Rome, Italie, FAO, $45 \mathrm{p}$.

15. METZGER R., CENTRES J.M., THOMAS L., LAMBERT J.C., 1995 L'approvisionnement des villes africaines en lait et produits laitiers. Un potentiel pour le développement rural. Rome, Italie, FAO, 105 p. (Etude $\left.\mathrm{n}^{\circ} 124\right)$

16. PONCE P., CAPDEVILLA J., ALFONSO H.A., LOPEZ M.G., LEON R., TABOADA A., 2003. Conservación de la leche en Cuba mediante la activación del sistema lactoperoxidasa. La Habana, Cuba, Centro Nacional de Sanidad Agropecuaria, CENSA, 33 p.

Accepté le 14.09.2009 


\section{Summary}

Lhoste F. Limits of the Application of the Method of Raw Milk Preservation by the Lactoperoxidase System in West Africa

In this study were analyzed the reasons why adopting the method of raw milk preservation by the lactoperoxidase system (LP-s) in African countries did not give the expected results, despite the interest in the method. The analysis was based on the assessment of the impact of the Bush Milk project launched by the FAO in 2001 on project evaluation missions, which were carried out in three countries of Western Africa, and on a regional workshop held in Niger in 2004. The main limit to adopting this method is related to the lack of professional organizations in local dairy chains, essential in the application of the LP-s method.

Keywords: Milk collection - Preservation - Codex Alimentarius West Africa.

\section{Resumen}

Lhoste F. Límites de aplicación del método de conservación de la leche cruda mediante el sistema lactoperoxidasa en África del Oeste

En este estudio se analizaron las razones por las cuáles la adopción del método de conservación de la leche cruda mediante el sistema de lactoperoxidasa (s-LP) en los países africanos tuvo resultados mitigados a pesar del interés que suscitó. El análisis se basó en la evaluación del impacto del proyecto de Leche de zonas no urbanas lanzado en 2001 por la FAO y se apoyó en la misión de evaluación del proyecto, llevada en tres países de África del Oeste, así como sobre el taller regional, Ilevado a cabo en Níger en 2004. La principal limitación en la adopción de este método estuvo ligada a la falta de estructuración profesional de las filiales lecheras locales, necesaria para la utilización del método s-LP.

Palabras clave: Recogida de leche - Preservación - Codex Alimentarius - Africa Occidental. 